\title{
Fatal pulmonary infection due to Mycobacterium fortuitum
}

\author{
M P A Lessing, $M M$ Walker
}

\begin{abstract}
Environmental (atypical, opportunist, other) mycobacteria were first isolated nearly a century ago. The classification of these "other than Mycobacterium tuberculosis" organisms was initially chaotic until Runyon proposed a scheme of four groups in 1959. Mycobacterium fortuitum is a member of group IV: Rapid growers. These ubiquitous terrestrial and aquatic forms contaminate water supplies, reagents, and clinical samples. They may colonise the respiratory systems of patients whose local defence mechanisms have been impaired or those with congenital and acquired immune defects. They can also cause disease in immunocompetent individuals.
\end{abstract}

There have been fewer than 20 published cases of pulmonary infection caused by $M$ fortuitum. A further case is reported of fatal pulmonary infection in an elderly patient with long standing chronic obstructive airways disease (COAD). He had left upper zone shadowing on chest radiography and lung abscesses at post mortem examination yielded only $M$ fortuitum.

(F Clin Pathol 1993;46:271-272)

Pathogenic mycobacteria are clinically divided into three groups; the two major pathogens Mycobacterium tuberculosis and $M$ leprae, the environmental species definitely associated with disease, and those rarely so. ${ }^{1}$ The environmental mycobacteria are also known as atypical, opportunist, or mycobacteria other than $M$ tuberculosis, none of which are completely satisfactory terms. There are four main types of disease caused by these opportunists: pulmonary disease, disseminated disease, which is particularly associated with HIV infection, lymph node disease in children, and local skin and soft tissue infections. ${ }^{1}$ We discuss the bacteriology of $M$ fortuitum and present the clinico-pathological findings of a patient from whom the organism was isolated and proved fatal.

\section{Case report}

An 80 year old man with a 10 year history of smoking related chronic obstructive airways disease (COAD) was admitted, complaining of shortness of breath on minimal activity and sweats. Nine years earlier he had undergone an abdominoperineal resection of an adeno- carcinoma of colon (stage Dukes A), thought to be cured. On examination he appeared tachypnoeic, pink, and cushingoid but was afebrile with an irregular pulse of $80 /$ minute, blood pressure $150 / 80 \mathrm{~mm} \mathrm{Hg}$ and a peak expiratory flow rate of $85 \mathrm{1} /$ minute. The breath sounds were distant and further clinical examination was unremarkable apart from a colostomy stoma noted.

Investigations showed a haemoglobin concentration of $17 \cdot 8 \mathrm{~g} / \mathrm{dl}$, packed cell volume $0.50 \mathrm{l} / 1$, white cell count $13.5 \times 10^{9} / 1(87 \%$ polymorphonuclear cells), and a platelet count $622 \times 10^{9} / 1$. The arterial blood gas result on room air was pO2 $8.2 \mathrm{kPa}(62 \mathrm{~mm} \mathrm{Hg}$ ), pCO2 $5.0 \mathrm{kPa}(38 \mathrm{~mm} \mathrm{Hg})$, serum bicarbonate $30 \mathrm{mmol} / \mathrm{l}$ and $\mathrm{pH} 7 \cdot 5$. A resting 12-lead electrocardiogram showed a few supraventricular ectopics. The chest radiograph confirmed overinflated lung fields. Clinical diagnosis was of exacerbation of COAD and he was treated with a nebulised bronchodilator, amoxycillin and erythromycin, and his maintenance low dose prednisolone was increased to $30 \mathrm{mg}$ daily with little benefit. Repeat chest radiography showed extending left upper and middle zone shadowing, and a differential diagnosis of an atypical pneumonia, pulmonary tuberculosis, or neoplasia was considered. Auramine staining for acid fast bacilli was negative on a single sputum sample which was inadequate for cytology. The patient deteriorated and died before further effective measures could be taken.

At post mortem examination there were two abscesses $8 \mathrm{~cm}$ and $6 \mathrm{~cm}$ in diameter in the left upper lobe. The remaining lung fields were oedematous and the right lower lobe consolidated. Histological examination of the abscesses showed caseating granulomata with abundant acid fast bacilli present on ZiehlNeelsen staining. $M$ fortuitum was isolated from the lung abscess material, identified by the Mycobacterium Reference Laboratory (PHLS).

\section{Discussion}

Runyon described the four groups of other mycobacteria according to pigment production and rate of growth in $1959 .{ }^{2}$ Group IV, the rapid growers, which may be photo-, scoto-, or more usually nonchromogenic, are defined as visible growth on LöwensteinJensen medium within seven days ${ }^{2}$ on subculture (primary culture of clinical material can take much longer). Küster (1905) described the frog tubercle bacillus which Bergey et al (1923) raised to species status as $M$ ranae 
Laboratory identification of Mycobacterium fortuitum

\begin{tabular}{|c|c|c|c|c|c|c|c|c|}
\hline Organism & Pigment & $\begin{array}{l}\text { Arylsulphatase } \\
\text { after } 3 \text { days }\end{array}$ & $\begin{array}{l}\text { Nitrate } \\
\text { reduction }\end{array}$ & $\begin{array}{l}\text { Iron } \\
\text { uptake }\end{array}$ & $\begin{array}{l}5 \% \\
\mathrm{NaCl}\end{array}$ & $\begin{array}{l}\text { Sodium } \\
\text { nitrate }\end{array}$ & Mannitol & Inositol \\
\hline \multicolumn{9}{|l|}{$M$ fortuitum biovariant } \\
\hline fortuitum & - & + & + & + & + & - & - & - \\
\hline peregrinum & - & + & + & + & + & - & + & - \\
\hline "third group" (unnamed) & - & + & + & + & + & - & + & + \\
\hline
\end{tabular}

(Latin rana; a frog). da Costa Cruz (1938) named an isolate from a post injection abscess $M$ fortuitum which was later established by Stanford and Gunthorpe (1969) to be the same organism as that originally described as $M$ ranae. $M$ fortuitum became common usage and Runyon (1972) recommended that the epithet ranae be discarded in favour of fortuitum. ${ }^{3}$ The Judicial Commission of the International Association of Microbiological Societies ruled in support of the recommendation. The $M$ fortuitum complex consists of the $M$ fortuitum biovariants and $M$ chelonae subspecies. ${ }^{4}$

Diagnostic bacteriology laboratories in England and Wales refer mycobacterial isolates to specialist reference centres for identification. Woods et al noted that $M$ fortuitum is the only mycobacterium which does not stain with the commonly used auramine-rhodamine fluorescence microscopy technique but we cannot confirm this observation. ${ }^{4}$ There is no universally accepted identification scheme for these organisms, ${ }^{5}$ with each laboratory relying on cultural characteristics (growth rate, temperature, pigment production), biochemical reactions, and antimicrobial sensitivity patterns. The table gives the diagnostic criteria for $M$ fortuitum, which may also be phenotypically divided into three biovariantsnamely, fortuitum, peregrinum, and a "third group" (unnamed).$^{6}$ Thin layer chromatography of cell wall lipids, mycolic acid detection by gas liquid chromatography linked with mass spectrophotometry, and other sophisticated methods have also been described. ${ }^{5}$

Dramatic outbreaks of $M$ fortuitum prosthetic valve endocarditis and sternal wound infections aroused interest in these environmental mycobacteria. ${ }^{6}$ The incidence of this disease is unknown and depends on the opportunity for transmission and host susceptibility. These are unaffected by public health measures because person-to-person spread rarely, if ever, occurs. ${ }^{4}$ An eight year survey of positive cultures referred from the south east of England to the Dulwich Public Health Laboratory Regional Tuberculosis Centre London (1977-84) showed that of 533 clinically confirmed new cases of opportunist mycobacterial infections, $8 \%$ were due to the rapid growers. At St Mary's Hospital, in the three year period June 1989-May 1992, a total of 5985 pooled samples were received for mycobacterial investigation. Tuberculosis was diagnosed in 123 patients and 13 patients grew $M$ fortuitum although the clinical importance of the latter was not confirmed.

Skin and soft tissue infections have been well documented, ${ }^{146}$ but less than 20 cases of pulmonary infection have been described. ${ }^{7-9}$ The route of entry is by inhalation of natural aerosols. Patients with impaired local lung defence mechanisms such as COAD, pneumoconiosis, and cystic fibrosis, or acquired and congenital immune defects are at risk, but patients without these risk factors have also been reported. ${ }^{79}$ The presentation of pulmonary infection includes bronchopneumonia, lung abscess, empyema, and a case of an asymptomatic enlarging pulmonary nodule has also been described. ${ }^{79}$ Our case report discusses an elderly male patient who had a long history of $\mathrm{COAD}$ requiring steroids and complicated by lung abscesses.

$M$ fortuitum pulmonary disease is diagnosed by isolation and identification of the organism but remains tendentious because of these organisms' ubiquity and their contaminating and colonising potential. ${ }^{156}$ The American Thoracic Society's Diagnostic Standards and Classification of Tuberculosis and Other Mycobacterial Diseases (14th edition) lists the requirements for making the diagnosis: (1) evidence of disease, such as an infiltrate on chest radiograph and no other cause found; and (2) repeated isolation of multiple colonies of the same isolate, or from a closed lesion such as an abscess or biopsy material.

The natural history of pulmonary disease due to rapidly growing mycobacteria is poorly defined but seems to be progressive, not responding to conventional antituberculosis treatment. Recommended treatment includes initial amikacin-cefoxitin combination, ${ }^{4}$ but newer agents such as the fluoroquinolones (ciprofloxacin, ofloxacin) ${ }^{9}$ and carbapenems (imipenem) ${ }^{4}$ have been successfully used as well as surgery. Deaths seem to be rare but a fatal case reported by the Brompton Hospital London in $1984,{ }^{8}$ together with others since and our report, confirm the real threat which $M$ fortuitum poses, especially to patients with pulmonary disease or immune defects.

We thank the following: Dr PA Jenkins of the Mycobacterium Reference Unit, Public Health Laboratory, Cardiff, for the identification of the isolate; $\operatorname{Dr} S$ Roche, consultant in medicine for the elderly, for permission to publish; and $\mathrm{Mr} M$ Savage for support

1 Grange JM, Yates MD. Infections caused by opportunis mycobacteria: a review $\mathcal{f} R$ Soc Med 1986;79:226-9.

2 Runyon EH. Anonymous mycobacteria in pulmonary disease. Med Clin North Am 1959;43:273-90.

3 Runyon EH. Conservation of the specific epithet fortuitum in the name of the organism known as Mycobacterium fortuitum da Costa Cruz. Int 7 Syst Bacteriol 1972 22:50-1.

4 Woods GL, Washington JA. Mycobacteria other than Mycobacterium tuberculosis: review of microbiologic and clinical aspects. Rev Infect Dis 1987;79:275-9.

5 Jenkins PA. The laboratory diagnosis of mycobacterial disenkins PA. The laboratory diagnosis of

6 Wallace RI Jr, Swenson JM, Silcox VA, et al. Spectrum of disease due to rapidly growing mycobacteria. Rev Infect disease due to rapidly

7 Pesce RR, Fejka S, Colodny SM. Mycobacterium fortuitum presenting as an asymptomatic enlarging pulmonary nodule. Am ₹ Med 1991;91:310-12.

8 Efthimiou J, Smith MJ, Hodson ME, et al. Fatal infection with Mycobacterium fortuitum in cystic fibrosis. Br $\mathcal{F}$ Dis Chest 1984;78:299-301.

9 Vadakekalam J, Ward MJ. Mycobacterium fortuitum lung abscess treated with ciprofloxacin. Thorax 1991;46: 737-8. 